\title{
Myocardial infarction caused by pharmacological substances - case description and literature review
}

\author{
Tomasz Kameczura, Leszek Bryniarski, Sławomir Surowiec, Maryla Kocowska, Kalina Kawecka-Jaszcz, \\ Danuta Czarnecka
}

I Department of Cardiology, Interventional Electrocardiology and Hypertension, Jagiellonian University Medical College, Krakow, Poland

Postep Kardiol Inter 2013; 9, 3 (33): 250-255

DOI: $10.5114 /$ pwki.2013.37504

\begin{abstract}
Myocardial infarction (MI) is most commonly caused by atherosclerosis and/or inflammatory processes of coronary artery walls. The consequence of those phenomena is instability of the atherosclerotic plaque, activation of the coagulation cascade and thrombus formation which occludes the lumen of the vessel. Vasospasm and microembolisation may participate in MI pathogenesis. In young individuals with diagnosis of MI, coronarography often reveals no pathologies. Is reported that MIs without significant changes of the coronary arteries occur in $1 \%$ to $12 \%$ of patients. In this article we focus on chemical substances, medicines among them, which can be a cause of MI.
\end{abstract}

Key words: myocardial infarction, myocardial infarction causes, pharmacotherapy.

\section{Myocardial infarction in the course of seminoma chemotherapy}

We describe the case of a 39-year-old man, with no previous history of ischemic heart disease and no cardiovascular risk factors. The patient was admitted to the clinic because of ST segment elevation myocardial infarction (STEMI) of the antero-lateral wall, which occurred during the cycle of chemotherapy of seminoma. Six weeks before, after diagnosing testicle neoplasm with metastases dissemination to retroperitoneal and supraclavicular lymph-nodes (T2N2M1S2 - degree of advancement IIla), orchiectomy was performed and the patient was qualified for chemotherapy with bleomycin $(0.4 \mathrm{U} / \mathrm{kg})$, etoposide $\left(100 \mathrm{mg} / \mathrm{m}^{2}\right)$ and cisplatin $\left(20 \mathrm{mg} / \mathrm{m}^{2}\right)$ (BEP). A week after the first cycle of BEP stenocardial chest pain occurred, which remained on admission to the cath lab. In ECG there was ST-segment elevation in antero-lateral leads. The patient was given aspirin (300 mg p.o.), clopidogrel (600 mg p.o.) and unfractionated heparin (5000 U i.v.). In coronarography there was an irregular, ulcerated, boundary atherosclerotic plaque found in the proximal part of the left anterior descending artery. The plaque was not significant, with haziness which may correspond to thrombus (Figure 1) with TIMI 3 flow [1, 2].

During coronarography chest pain resolved and ECG recording normalized. Because of the overall clinical picture, two-antiplatelet therapy was maintained and low molecular weight heparin was included (enoxaparin $2 \times 80 \mathrm{mg} /$ day s.c.) for 7 days. The coronarography performed after a week showed a normal picture of coronary arteries (Figure 2).

In echocardiography hypokinesis of the apex and basal segment of the antero-septal wall with ejection fraction of $65 \%$ was found. The patient was discharged with the recommendation of regular taking of aspirin, clopidogrel, $\beta$-blocker, angiotensin receptor blocker and statin, and directed to further chemotherapy. At present the patient has undergone three cycles of BEP, feels good and there is no presence of cancer cells in PET.

The BEP scheme used for the treatment of seminoma is approved in treatment of testicular tumors derived from germ cells. Cisplatin and etoposide in this protocol are administered for 5 days in cycles every 21 days, the number of cycles varying from 2 to 4 . Bleomycin is administered on days 1,8 and 15 of therapy. High effectiveness of BEP is accompanied by serious sides effects such as pulmonary fibrosis, neutropenia and neurotoxicity.

\section{Medicines and chemical substances that can cause myocardial infarction}

Some drugs and chemical substances can cause MI, and their mechanisms can be diametrically different: from a di-

\section{Corresponding author:}

Prof. Leszek Bryniarski MD, PhD, I Department of Cardiology, Interventional Electrocardiology with Hypertension, Jagiellonian University Medical College, 17 Kopernika St, 31-501 Krakow, Poland, tel.: +48 1242473 00, e-mail: I_bryniarski@poczta.fm Received: 27.04.2013, accepted: 28.06.2013. 


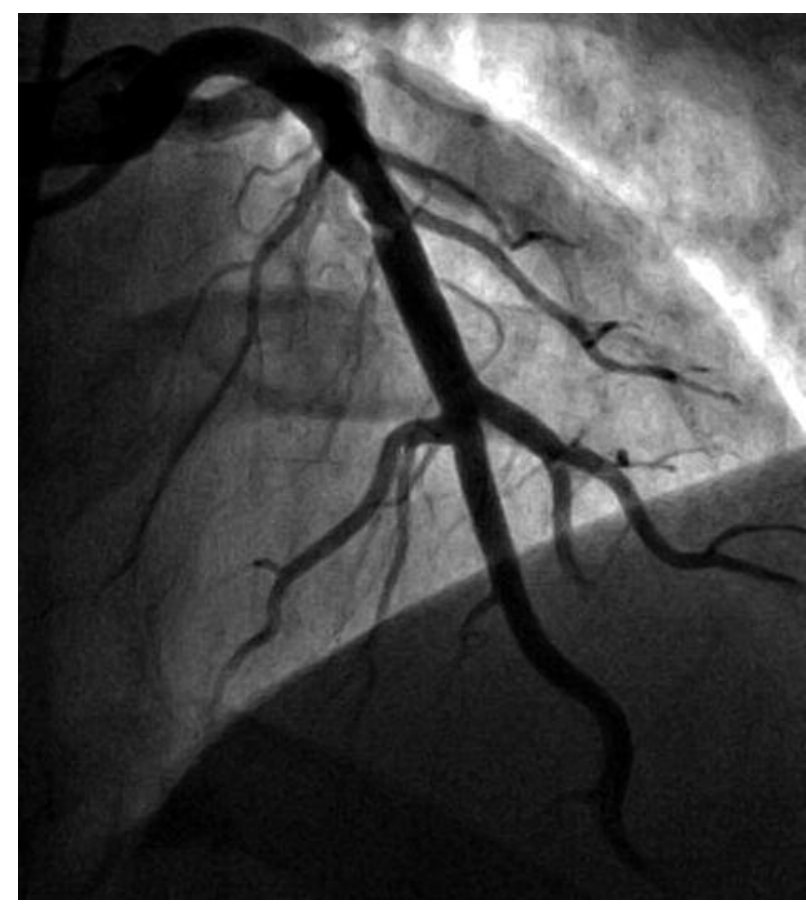

Fig. 1. Atherosclerotic plaque in left anterior descending artery

rect toxic effect on cardiomyocytes to probably the most common mechanism, which is vasospasm. There are cases of MI caused by chemotherapeutics [3], antimigraine drugs (triptans) [4], antibiotics (amoxycillin with clavulanic acid, cefuroxime) $[5,6]$ bromocriptine, $\beta$-blockers, pseudoephedrine (common supplement of antitussive medications) [7], acetylsalicylic acid (ASA), steroidal and non-steroidal antiinflammatory drugs [8], allopurinol, drugs inhibiting appetite and tyrosine. Also documented is the risk of $\mathrm{MI}$ after ingestion of such substances as ecstasy, methamphetamine, LSD, cocaine, heroin [7] and volatile substances (propanebutane) [9].

\section{Mechanisms of drug cardiotoxicity and other chemical substances}

Anti-cancer drugs: cisplatin and 5-fluorouracil

At greater risk of developing cardiovascular complications during treatment with cisplatin and 5-fluorouracil (5-FU) are patients with a history of ischemic heart disease and numerous cardiovascular risk factors. Harmfulness of those drugs comes from the effects they cause at systemic, tissue, cellular and mitochondrial levels [10]. Harmful effects of drugs can result from direct action on membrane receptors, second messenger system, channels and ion pumps and also on intracellular organelles [11]. By an action of modifying the morphology of ion channels drugs can influence polarization and depolarization of cardiomyocytes, leading to arrhythmias or impairment of left ventricular systolic and diastolic function. It points to drug interaction with

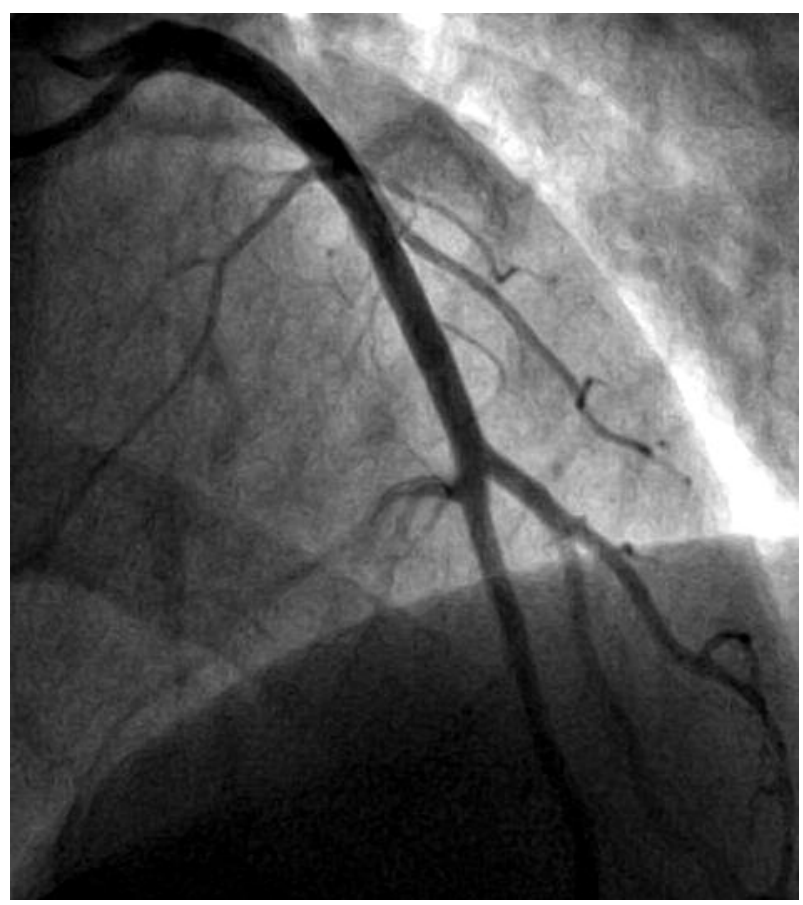

Fig. 2. Control coronary angiography

potassium channel h-ERG in cardiomyocytes. Drugs from the antidepressant tricyclic group inhibit this channel indirectly, contributing to the prolongation of QT interval and increased risk of malignant arrhythmia [11, 12]. Also endothelial damage caused by chemotherapeutics is known as a risk factor for myocardial infarction [13]. In the case of cisplatin different pathomechanisms can lead to MI. This alkylating cytostatic activates platelet aggregation, raises the production of thromboxane and activates the arachidonic acid pathway [14]. It also increases the synthesis of tumor necrotizing factor (TNF). As a result of tubular damage, cisplatin can cause hypomagnesemia, which sensitizes the vessels to noradrenaline, acetylcholine, serotonin, angiotensin, and potassium, and thus can cause spasm of the arteries [10]. During the treatment with 5-FU clinical symptoms of cardiotoxicity (ischemic changes in ECG, chest pain, MI, sudden cardiac death) occurred, according to data from retrospective studies, in from $1.6 \%$ to $10.2 \%$ of patients [15]. In a prospective study, Rezkalla et al. showed changes in the ST segment (elevations and depressions more than $1 \mathrm{~mm}$ ) in $68 \%$ of patients who underwent intravenous infusion of 5-FU. In those patients ischemic changes were asymptomatic but after the end of infusion two sudden deaths were recorded in the 25-patient study group [16]. Another study showed that the treatment with 5-FU and cisplatin was associated with occurrence of supraventricular arrhythmias (53\%), ventricular arrhythmias (47\%), and in $31 \%$ of patients ST segment deviation such as elevation and depression was observed [17]. According to the dose and the dura- 
tion of treatment subsequent ECG findings are observed: ST-segment elevation, negative T wave in precordial leads and high pointed T wave. Overall mortality in the course of 5 -FU treatment was assessed in different studies from $2.2 \%$ to $13 \%$, and a higher incidence of complications correlated with higher dose of the drug $\left(>800 \mathrm{mg} / \mathrm{m}^{2}\right)$ and continuous infusion compared to boluses. Complications most commonly occurred in 2 to 5 days after 5-FU infusion [14].

5-Fluorouracil impairs the body's antioxidant defense at the cellular level, thus intensifying lipid peroxidation and subsequent damage to the endothelium. It also negatively interferes with the production of nitric oxide [18]. In addition, it leads to DNA damage, blocking of ATP and release of proapoptotic proteins. Experimental studies showed that 5-FU causes dose-dependent and time-dependent depletion of high-energetic phosphates and citrate accumulation in the myocardium [19]. In pathogenesis of acute ischemia of myocardium disorders of coagulation and fibrinolysis can be involved [20]. During 5-FU therapy an increase in fibrinopeptide $A$ (FPA) and a decrease in tissue plasminogen activator (t-PA) were observed [21]. 5-Fluorouracil can also cause a coronary artery spasm. Reference is made here to impairment of nitric oxide synthase as well as endothelium-independent vasoconstriction mediated by protein kinase $\mathrm{C}$. The coronary artery spasm may also occur under the influence of a pro-drug of 5-FU - capecitabine.

Particularly exposed to the risk of such complications are patients with coronary heart disease in their history. Such a relationship may be due to the expression of thymidine phosphorylase in atherosclerotic plaques; this enzyme, present in high doses within the tumor, is responsible for conversion of 5'-deoxy-5-fluorouridine (5'-DFUR) (capecitabine metabolite) to 5-FU [19, 22].

\section{Non-steroidal anti-inflammatory drugs}

Cardiovascular complications in use of non-steroidal antiinflammatory drugs (NSAIDs) are mostly due to endothelial cell damage [23]. It is a well-known adverse effect of selective inhibitors of cyclo-oxygenase-2 (COX-2) such as rofecoxib, which caused a five-fold rise of cardiovascular risk [24]. Efficiency of coxibs in inflammatory disease treatment is comparable to NSAIDs but with reduced side-effects from the gastrointestinal tract. However, because of the significant increase of cardiovascular risk in patients treated with rofecoxib, it was withdrawn from the market. Adverse effects could be precisely due to selective COX-2 inhibition. Cyclooxygenase-2 is mostly responsible for development of inflammation. After in vitro research it was suggested that laminar shear stress in endothelial cells causes expression of COX-2, which becomes a source of anti-aggregative prostaglandin $\mathrm{I}_{2}\left(\mathrm{PGI}_{2}\right)[24,25]$. Furthermore, COX-2 inhibition may activate the lipoxygenase pathway and consequently intensify production of leukotrienes, stimulating coronary vasospasm. Due to weaker inhibition of COX-2 rel- ative to COX-1, celecoxib as a less selective inhibitor of COX2 should be safer compared to selective coxibs. It is still used in treatment of inflammation and pain, in rheumatic disorders and in patients with familial adenomatous polyposis. Nevertheless, in some studies in patients treated with celecoxib, a dose-dependent increase of cardiovascular risk (including myocardial infarction) was revealed [26]. To sum up, unfavorable effects of NSAIDs, both non-selective and selective for COX-2, are multipoint; therefore it is difficult to indicate the most significant mechanism. It appears that their influence on endothelium is crucial.

\section{Antiretroviral therapy}

Despite the efficiency of combined antiretroviral therapy in HIV-positive patients, many side-effects are observed. It was proven, in the multicenter prospective study of the DAD workgroup, that Highly Active Antiretroviral Therapy (HAART) caused increase of MI risk by about $26 \%$ per year of HAART. Age, smoking status (currently or in the past), history of cardiovascular diseases and male sex are independent risk factors in these patients [27]. The mechanism of action at cell level during HAART is due to the rising concentration of reactive oxygen species (ROS). These particles are side-products of metabolic reactions which occur in mitochondria. Intracellular level of ROS higher than antioxidative abilities of the cell causes damage of macromolecules such as lipids, proteins and DNA. Cardiomyocyte apoptosis caused by zidovudine is initiated by caspase- 3 and -7 and ADP-ribose polymerase concentrations, and also depends on the concentration of mitochondrial ROS [28]. In HIV-positive patients treated with abacavir myocardial infarction risk is rising [29]. Among nucleoside reverse transcriptase inhibitors (NRTI) abacavir, and in a lower degree didanosine, increase incidence of $\mathrm{Ml}$ among patients currently treated with them and also a few months after the drug's cessation. In other drugs in the same pharmacological group there were no similar observations (neither stavudine nor zidovudine). A significant rise of MI incidence among HIVpositive patients was observed after introduction of protease inhibitor $(\mathrm{PI})$ on the market, and also treatment with $\mathrm{PI}$ was an independent risk factor of $\mathrm{MI}$ [30]. During therapy with PI development of lipodystrophy, hyperlipidemia, hyperglycemia and insulin resistance is possible even in $60 \%$ of patients. These processes undoubtedly increase cardiovascular risk [31]. Binding of PI with LDL-related protein (LRP) impairs chylomicron uptake in the liver and endothelial triglyceride clearance, leading to hyperlipidemia and insulin resistance [32]. HIV infection could cause many unfavorable changes in the cardiovascular system, e.g. vasculitis (polyarteritis nodosa, Henoch-Schönlein purpura), druginduced hypersensitivity vasculitis [33], Takayasu's disease [34], and Kawasaki's disease-like syndrome [35]. Unfavorable effects of HIV infection on the cardiovascular system are presumably due to damage and dysfunction of endothelium [36], and also action of HIV-infected macrophages 
stimulating leukocyte adhesion or intensifying arteritis, and in this way increasing atherosclerosis progression. Coronary arteries are damaged by activation of cytokines, adhesive molecules and also changes in class I major histocompatibility complex on the surface of the smooth-muscle cell [32]. It is not inconceivable that viral glycoprotein gp120 induces apoptosis of the smooth-muscle cells [37]. Abnormality in coagulation (increased concentrations of D-dimer, plasminogen activator inhibitor-1, tissue plasminogen activator antigen) was also observed [31].

\section{Drugs}

Complications due to drugs and other psychoactive substances are still an increasing problem. It is proven that $41 \%$ of American adolescents are marihuana smokers, $13 \%$ of teenagers use ecstasy, $11 \%$ methamphetamine, 10\% LSD, $9 \%$ cocaine and $4 \%$ heroin [7]. Cocaine users in Europe are estimated at 12 million people [38]. Mechanisms which lead to cardiac complications after cocaine, amphetamine or ecstasy use are: vessel damage, prothrombotic effect and direct myocardial injury [39]. Furthermore, increase of oxygen consumption as a consequence of higher blood pressure and heart rate in a situation of simultaneous coronary artery spasm may lead to acute ischemia of cardiac muscle. As in the case of above-described medication groups, endothelial dysfunction (increase of endothelin-1 production, reduction of expression and production of nitric oxide synthase) and also prolonged vasospasm cause ischemia and can lead to MI. Risk of acute coronary syndrome is 24 -fold higher in a drug user within the $1^{\text {st }} \mathrm{h}$ after use than in people who are not on drugs.

\section{Carbon monoxide}

Carbon monoxide poisoning is one of the most common causes of hospitalization on ER departments in western countries. Clinical symptoms and the course of poisoning poorly correlate with the serum level of carboxyhemoglobin. The most common symptoms are: cardiotoxicity, such as malignant arrhythmias, ECG ischemic changes and MI. Also there was observed rapid pulmonary edema [40]. The mechanism leading to such drastic complications is higher affinity to hemoglobin molecules than $\mathrm{CO}$ molecules. Consequently, there is an exchange of oxygen molecules of the compounds of $\mathrm{O}_{2}-\mathrm{Hg}$. The resulting carboxyhemoglobin is ineffective when it comes to the delivery of oxygen to tissues and leads to ischemia [41]. Mechanisms mentioned in the literature that may be a cause of a heart attack due to exposure to $\mathrm{CO}$ include: the blocking of the main bonds of hemoglobin, which is an oxygen carrier, toxic effects on the mitochondria by the formation of a ligand of cytochrome a, a3-CO, which paralyzes the socalled mechanisms of cellular respiration, and eventually CO may cause thrombus formation in the coronary arteries, both in patients with atherosclerosis and in those without atherosclerosis [42]. In in vitro studies in mice, Stein et al. demonstrated the cytoprotective effect and the antiapoptotic effect of $\mathrm{CO}$ molecules on the myocardium. By the utilization donor molecule CO-CORM-3, in the mouse organism there is observed a late defense reaction against $\mathrm{MI}$. It involves a cascade of transcription including factors nuclear factor $\kappa \mathrm{B}(\mathrm{NF}-\kappa \mathrm{B}), \mathrm{STAT1} / 3$ and Nrf2 with subsequent increase of cardio-protective and anti-apoptotic substances in myocardium [43].

\section{Appetite suppressants}

Among medications which inhibit the hunger center [44] unfavorable effects were observed in treatment with phentermine and sibutramine. Phentermine works on the hypothalamus to stimulate adrenal glands to release norepinephrine, a chemical messenger that signals the fight-or-flight response and reduces hunger. Sibutramine is a centrally acting serotonin-norepinephrine reuptake inhibitor. Side-effects of these medications are strongly expressed and related to excessive sympathetic activation. They include increase of heart rate, myocardial contractility, conduction and also a vasoconstrictive effect [45]. Increased incidence of MI and stroke was observed among patients treated with these appetite suppressants. Phentermine is not registered in Poland and sibutramine has been withdrawn from the market.

\section{Spasm of coronary artery}

One of the causes of acute coronary syndrome and arrhythmias in young people can be a coronary artery spasm [7]. As mentioned above, the frequency of myocardial infarction in patients with a normal coronary artery is assessed at $1-12 \%$ and in the group of patients at age below 35 years the rate reaches $20 \%$. In this population, in teenagers and young adults, there is observed an increasing number of MIs induced by stimulants and narcotics. The most frequently used are alcohol, marijuana, nicotine and cocaine.

These substances exaggerate endothelial dysfunction, intensify platelet aggregation and sympathetic activity, and cause reduction of nitric oxide (NO) synthase activity in the vascular wall. A low level of basic nitric oxide secretion due to impairment of endothelial function and increased contractility due to higher kinase Rho/Rho activity are mentioned as the most important causes inducing vasospasm.

Among other factors predisposing to vasospasm are: increased sympathetic activity, oxidative stress, chronic inflammation and hypomagnesemia. Other factors which can lead to spasm of the coronary artery are genetic factors such as polymorphism of the gene responsible for synthesis of nitric oxygen synthase and synthesis of paraoxonase [46].

Another mechanism of spasm was described in the course of Kounis syndrome [47]. Kounis syndrome is a generalized allergic reaction, which can be triggered by medication and leads to degranulation of mast cells. These mast cells are localized in adventitia of coronary arteries and near 
the plaque. Kounis syndrome, characterized by sudden release of inflammatory markers such as histamine and proteolytic enzymes (chymase, tryptase), causes lysis of collagen covering the plaque. These reactions lead to thrombogenic material exposure and to activation factors of the coagulation cascade. Moreover, into the circulatory system are released other substances, such as products of arachidonic acid metabolism, cytokines, chemokines and platelet activating factor (PAF) [47-49]. Drugs which can induce Kounis syndrome are: antibiotics (ampicillin, amoxicillin, cefuroxime, penicillin, sulbactam, vancomycin), steroids (betamethasone, hydrocortisone), chemotherapeutics (5FU, sirolimus, paclitaxel), analgesics (etomidate, suxamethonium), non-steroidal anti-inflammatory drugs (acetylsalicylic acid, ibuprofen, metamizole), anticoagulants and fibrinolytics (heparin, streptokinase, urokinase). There were described symptoms of Kounis syndrome after use of allopurinol, enalapril, esmolol, omeprazole and contrast medium as well. Such complications were also observed as a reaction to a stent component (nickel) and drug-eluting stent (paclitaxel) [33, 34].

\section{Conclusions}

In patients without symptoms before the planned chemotherapy it is particularly important to assess the profile of coronary disease risk factors for prevention of heart attack. Higher cardiovascular risk of complications occurs among patients with a history of anaphylaxis. In patients with normal coronary arteries and history of heart infarction, due to a common cause of coronary instability which is coronary artery spasm, it is advisable to consider a test with ergonovine or acetylcholine. In such patients, the first choice treatment is the use of calcium channel blockers and nitrates. Another issue is the need for education, especially of young people, about the dangers of the use of drugs, including the possibility of causing a heart attack.

\section{References}

1. Van de Werf F, Bax J, Betriu A, et al. Management of acute myocardial infarction in patients presenting with persistent ST-segment elevation: the Task Force on the Management of ST-Segment Elevation Acute Myocardial Infarction of the European Society of Cardiology. Eur Heart J 2008; 29: 2909-2945.

2. Iuliano L, Micheletta F, Napoli A, Catalano C. Myocardial infarction with normal coronary arteries: a case report and review of the literature. J Med Case Reports 2009; 3: 24.

3. McGlinchey PG, Webb ST, Campbell NP. 5-fluorouracil-induced cardiotoxicity mimicking myocardial infarction: a case report. BMC Cardiovasc Disord 2001; 1: 3.

4. Weder CR, Schneemann M. Triptans and troponin: a case report. Orphanet J Rare Dis 2009; 4: 15.

5. Mazarakis A, Koutsojannis CM, Kounis NG, Alexopoulos D. Cefuroxime-induced coronary artery spasm manifesting as Kounis syndrome. Acta Cardiol 2005; 60: 341-345.

6. Del Furia F, Querceto L, Testi S, Santoro GM. Acute ST-segment elevation myocardial infarction complicating amoxycillin-induced anaphylaxis: a case report. Int J Cardiol 2007; 117: e37-e39.
7. El Menyar AA. Drug-induced myocardial infarction secondary to coronary artery spasm in teenagers and young adults. J Postgrad Med 2006; 52: 51-56.

8. Kumar A, Berko NS, Gothwal R, et al. Kounis syndrome secondary to ibuprofen use. Int J Cardiol 2009; 137: e79-e80.

9. Godlewski K, Werner B, Sterliński M, et al. Zawał serca u 14-letniego chłopca po inhalacji butanu. Kardiol Pol 2006; 64: 305-308.

10. Kinhult S, Albertsson M, Eskilsson J, Cwikiel M. Effects of probucol on endothelial damage by 5-fluorouracil. Acta Oncol 2003; 42: 304-308.

11. Tabrizchi R. Molecular mechanisms of adverse drug reactions in cardiac tissue. Handb Exp Pharmacol 2010; 196: 77-109.

12. Testai L, Bianucci AM, Massarelli I, et al. Torsadogenic cardiotoxicity of antipsychotic drugs: a structural feature, potentially involved in the interaction with cardiac HERG potassium channels. Curr Med Chem 2004; 11: 2691-2706.

13. Singh R, Sagar TG, Ramanan SG. 5-Fluorouracil cardio-toxicityrevisited. Indian J Med Pediatr Oncol 2004; 25: 35-38.

14. Yeh ET, Bickford CL. Cardiovascular complications of cancer therapy: incidence, pathogenesis, diagnosis, and management. J Am Coll Cardiol 2009; 53: 2231-2247.

15. Gradishar WJ, Vokes EE. 5-Fluorouracil cardiotoxicity: a critical review. Ann Oncol 1990; 1: 409-414.

16. Rezkalla S, Kloner RA, Ensley J, et al. Continuous ambulatory ECG monitoring during fluorouracil therapy: a prospective study. J Clin Oncol 1989; 7: 509-514.

17. Cappelaere P, Vincent A, Staumont M, et al. Continuous Holter cardiac monitoring and chemotherapy by combination with platinum and fluoro-uracil. Bull Cancer 1991; 78: 261-272.

18. Ramot Y, Nyska A. Drug-induced thrombosis: experimental, clinical, and mechanistic considerations. Toxicol Pathol 2007; 35: 208-225.

19. Frickhofen N, Beck FJ, Jung B, et al. Capecitabine can induce acute coronary syndrome similar to 5-fluorouracil. Ann Oncol 2002; 13: 797-801.

20. Steciwko A, Reksa D, Grotowska M. Endothelium and its role in pathogenesis of diseases. Pol Arch Med Wewn 2006; 116: 819-831.

21. Ruiz MA, Marugan I, Estelles A, et al. The influence of chemotherapy on plasma coagulation and fibrinolytic systems in lung cancer patients. Cancer 1989; 63: 643-648.

22. Camaro C, Danse PW, Bosker HA. Acute chest pain in a patient treated with capecitabine. Neth Heart J 2009; 17: 288-291.

23. Głuszko P, Bielińska A. Non-steroidal anti-inflammatory drugs and the risk of cardiovascular diseases: are we going to see the revival of cyclooxygenase-2 selective inhibitors? Pol Arch Med Wewn 2009; 119: 231-235.

24. Fitzgerald GA. Coxibs and cardiovascular disease. N Engl J Med 2004; 351: 1709-1711.

25. Grosser T, Fries S, FitzGerald GA. Biological basis for the cardiovascular consequences of COX-2 inhibition: therapeutic challenges and opportunities. J Clin Invest 2006; 116: 4-15.

26. Solomon SD, McMurray JJ, Pfeffer MA, et al. Cardiovascular risk associated with celecoxib in a clinical trial for colorectal adenoma prevention. N Engl J Med 2005; 352: 1071-1080.

27. Friis-Moller N, Sabin CA, Weber R, et al. Combination antiretroviral therapy and the risk of myocardial infarction. N Engl J Med 2003; 349: 1993-2003.

28. Gao RY, Mukhopadhyay P, Mohanraj R, et al. Resveratrol attenuates azidothymidine-induced cardiotoxicity by decreasing mitochondrial reactive oxygen species generation in human cardiomyocytes. Mol Med Report 2011; 4: 151-155. 
29. Obel N, Farkas DK, Kronborg G, et al. Abacavir and risk of myocardial infarction in HIV-infected patients on highly active antiretroviral therapy: a population-based nationwide cohort study. HIV Med 2010; 11: 130-136.

30. Holmberg SD, Moorman AC, Williamson JM, et al. Protease inhibitors and cardiovascular outcomes in patients with HIV-1. Lancet 2002; 360: 1747-1748.

31. Barbaro G, Fisher SD, Lipshultz SE. Pathogenesis of HIV-associated cardiovascular complications. Lancet Infect Dis 2001; 1: 115-124.

32. Barbaro G. HIV infection, highly active antiretroviral therapy and the cardiovascular system. Cardiovasc Res 2003; 60: 87-95.

33. Ho JE, Hsue PY. Cardiovascular manifestations of HIV infection. Heart 2009; 95: 1193-1202.

34. Shingadia D, Das L, Klein-Gitelman M, Chadwick E. Takayasu's arteritis in a human immunodeficiency virus-infected adolescent. Clin Infect Dis 1999; 29: 458-459.

35. Johnson RM, Little JR, Storch GA. Kawasaki-like syndromes associated with human immunodeficiency virus infection. Clin Infect Dis 2001; 32: 1628-1634.

36. Chi D, Henry J, Kelley J, et al. The effects of HIV infection on endothelial function. Endothelium 2000; 7: 223-242.

37. Twu C, Liu NQ, Popik W, et al. Cardiomyocytes undergo apoptosis in human immunodeficiency virus cardiomyopathy through mitochondrion- and death receptor-controlled pathways. Proc Natl Acad Sci U S A 2002; 99: 14386-14391.

38. Lucena J, Blanco M, Jurado C, et al. Cocaine-related sudden death: a prospective investigation in south-west Spain. Eur Heart J 2010; 31: 318-329.

39. Macmahon JM, Tallentire VR. Stimulating stuff: the pathological effects of cocaine and amphetamines on thecardiovascular system. BJDVD 2010; 10: 251-255.

40. Raub JA, Mathieu-Nolf M, Hampson NB, Thom SR. Carbon monoxide poisoning: a public health perspective. Toxicology 2000; 145 : $1-14$.

41. Lippi G, Rastelli G, Meschi T, et al. Pathophysiology, clinics, diagnosis and treatment of heart involvement in carbon monoxide poisoning. Clin Biochem 2012; 45: 1278-1285.

42. Varol E, Ozaydin M, Aslan SM, et al. A rare cause of myocardial infarction: acute carbon monoxide poisoning. Anadolu Kardiyol Derg 2007; 7: 322-323.

43. Stein AB, Bolli R, Dawn B, et al. Carbon monoxide induces a late preconditioning-mimetic cardioprotective and antiapoptotic milieu in the myocardium. J Mol Cell Cardiol 2012; 52: 228-236.

44. Azarisman SM, Magdi YA, Noorfaizan S, Oteh M. Myocardial infarction induced by appetite suppressants in Malaysia. N Engl J Med 2007; 357: 1873-1874.

45. Surowiec S, Jankowski P. Pharmacological treatment of obesity in adults. Pol J Cardiol 2007; 9: 221-225.

46. Kusama Y, Kodani E, Nakagomi A, et al. Variant angina and coronary artery spasm: the clinical spectrum, pathophysiology, and management. J Nippon Med Sch 2011; 78: 4-12.

47. Ridella M, Bagdure S, Nugent K, Cevik C. Kounis syndrome following beta-lactam antibiotic use: review of literature. Inflamm Allergy Drug Targets 2009; 8: 11-16.

48. Biteker M, Duran NE, Biteker FS, et al. Allergic myocardial infarction in childhood: Kounis syndrome. Eur J Pediatr 2010; 169: 27-29.

49. Kounis NG, Hahalis G, Theoharides TC. Coronary stents, hypersensitivity reactions, and the Kounis syndrome. J Interv Cardiol 2007; 20: 314-323. 